\title{
Experimental and numerical investigation on the asymmetry of the current density extracted through a plasma meniscus in negative ion accelerator
}

\author{
S. Denizeau ${ }^{1,6}$, D. Aprile ${ }^{1,5}$, G. Fubiani ${ }^{2}$, F. Taccogna ${ }^{3}$, P. Minelli ${ }^{3}$, M. Ichikawa ${ }^{4}$, J. \\ Hiratsuka ${ }^{4}$, M. Kashiwagi ${ }^{4}$, A. Kojima ${ }^{4}$, G. Chitarin ${ }^{1,6}$ \\ ${ }^{1}$ Consorzio RFX, Padua, Italy \\ ${ }^{2}$ LAPLACE, Université de Toulouse, CNRS, Toulouse, France \\ ${ }^{3}$ CNR-Institute for Plasma Science and Technology, Bari, Italy \\ ${ }^{4}$ QST, National Institutes for Quantum and Radiological Science and Technology, Naka, Ibaraki, Japan \\ ${ }^{5}$ INFN, Laboratori Nazionali di Legnaro, Padua, Italy \\ ${ }^{6}$ University of Padua, Padova, Italy
}

E-mail: sylvestre.denizeau@igi.cnr.it

Received xxxxxx

Accepted for publication $\mathrm{xxxxxx}$

Published xxxxxx

\begin{abstract}
In multi-beamlet negative ion accelerators for Neutral Beam Injectors, the transverse magnetic field necessary for suppressing the co-extracted electrons induces a deflection of the negative ion beamlets that must be corrected. For the design, particle-tracing simulation codes are normally used to compute the ion trajectories inside the acceleration stage, using simplified assumptions concerning the boundary conditions on the surface (called meniscus) where the negative ions are extracted from the plasma. Recently, experimental campaigns dedicated to the accurate measurement of the beamlet deflection revealed a higher deflection than the one foreseen by simulations under several operating conditions. In this work, we demonstrate that a much better agreement with the experimental data can be obtained by incorporating in the numerical simulations a non-radial symmetric distribution of the ion current density extracted across the meniscus surface. We also show that such asymmetric distribution is consistent with a Particle in Cell (PIC) simulation of the plasma source in the presence of transverse magnetic field.

In the first part of this work, the asymmetry of the ion current density at the meniscus is studied in an empirical way, by analysing and fitting the experimental results obtained for different values of the operating parameters. In the second part, a PIC simulation of the ion source allows to evidence and quantify the asymmetry of the $\mathrm{H}^{-}$ion flow in the meniscus zone.
\end{abstract}

Keywords: Neutral Beam Injector, negative ion accelerator, beam extraction, beam deflection, current density asymmetry, asymmetric meniscus, particle-in-cell 


\section{Introduction:}

In Neutral Beam Injectors (NBIs) for fusion experiments, several hundreds of negative ion beamlets are extracted from a plasma source and accelerated in an electrostatic accelerator, to produce a single negative ion beam. The ion beam is then neutralized to obtain a neutral beam having the required power and aiming. A transverse magnetic field is necessary in the plasma source, for filtering the high-energy electrons and facilitating the production of negative ions, and in the extraction region of the accelerator, to deflect and suppress the residual electrons co-extracted with the negative ions. However, the transverse magnetic field also produces an undesired deflection of each ion beamlet, resulting in a divergence of the whole beam. This divergence must remain low to ensure a good efficiency of the beam neutralization and transport to the tokamak [1]. Several solutions have been developed to minimize the deflection of the beamlets (steering grid, special magnetic configuration [2]...). For NBIs, the compensation for the beamlet deflection must be guaranteed by design, before starting beam operation, as subsequent corrections are complex and timeconsuming. It is thus necessary to compute the ion beam trajectory from the meniscus to the exit of the accelerator, in three dimensions with very accurate raytracing codes such as Opera [3]. However, these simulations rely on simplifying assumptions on the properties of the plasma meniscus, which is the locus where ions are extracted from the plasma, in the vicinity of each aperture. In fact, due to the difficulties in modelling or directly measuring the current density at the meniscus, a uniform distribution is usually assumed as boundary condition in the simulations, even though the motion of the negative ions is likely to be affected by the transverse magnetic field effect on the plasma in the extraction region.

In 2016 and 2017, a collaboration between Consorzio RFX (Padua, Italy) and QST (Naka, Ibaraki, Japan) was dedicated to the measurement of the beamlet deflection under various operating conditions on the Negative Ion Test Stand (NITS) in the QST lab in Naka [4][5]. In particular, several experiments were jointly carried out using specifically defined magnetic configurations [2] in order to accurately evaluate the beamlet deflection caused by the transverse magnetic field and to achieve its complete compensation.

The data obtained during the joint experimental campaigns evidenced that the beamlet deflection was underestimated in the simulations, as already observed in previous experimental results [6].

It is important to understand the cause of such discrepancy and to rectify it in future simulations, otherwise the design of any deflection correction system might be inevitably wrong. Based on several previous works [7][8][9][10][11][12], we presume that the underestimated deflection obtained from simulations is a consequence of having assumed a radially symmetric ion current density distribution within each meniscus, thus disregarding the following physical phenomena:

1. in the plasma source in the vicinity of meniscus, a transverse transport of the negative ions is caused by the transverse magnetic field [9];

2. this phenomenon results in a non-radial symmetric negative ion current density through the plasma meniscus which is formed just upstream of each aperture;

3. the non-radial symmetric extracted current density causes a non-uniform space-charge distribution inside each beamlet and thus an additional deflection with respect to an ideal symmetric beamlet.

In this study we demonstrate that, by correctly evaluating these three phenomena, we can obtain an evaluation of the beamlet deflection in good agreement with the experimental results in quite a large range of operating parameters.

In the first part of this work, we assume a simple circular asymmetric meniscus, having a higher current density on one half than on the other half. The asymmetry of this current density profile is quantified by a figure of merit $\alpha_{u n b}$ used as initial condition in the beamlet trajectory simulations. This asymmetry factor $\alpha_{u n b}$ is then adjusted so that the beamlet deflection in the simulations matches the experimental measurements.

In the second part of the work, a 3D Particle in Cells simulation of a scaled down plasma source and extractor is used to determine the motion of the ions and a realistic profile of the extracted current density, in the presence of transverse magnetic field in the plasma source. In these simulations, the asymmetry of the current distribution is indeed observed, with an order of value of $\alpha_{u n b}$ consistent with the ad hoc assumptions made in the first part of this paper.

\section{Experimental study of the beamlet deflection, joint QST-RFX experiments on NITS}

\subsection{Experimental set-up}

NITS is a negative ion beam accelerator with a plasma source shaped as a truncated cylinder, known as kamaboko. The plasma is produced by hot tungsten filaments positioned around the cylinder and confined by a multi-cusp shaped magnetic field produced by permanent magnets located all around the cylinder. The extraction area is located on the front planar side. Inside the source, a magnetic field transverse to the extraction direction - the Filter Field - is used to reduce the electron velocity between the filaments and the 
extraction area in order to minimize the ratio of coextracted electrons. The acceleration stage comprises three grids: the Plasma Grid (PG), through which the beam is extracted, the Extraction Grid (EG) and the
Grounded Grid (GG). Each grid has two groups of $3 \times 5$ apertures. The grid geometry and acceleration gaps are designed to reproduce the MITICA/ITER HNB accelerator configuration. (a)

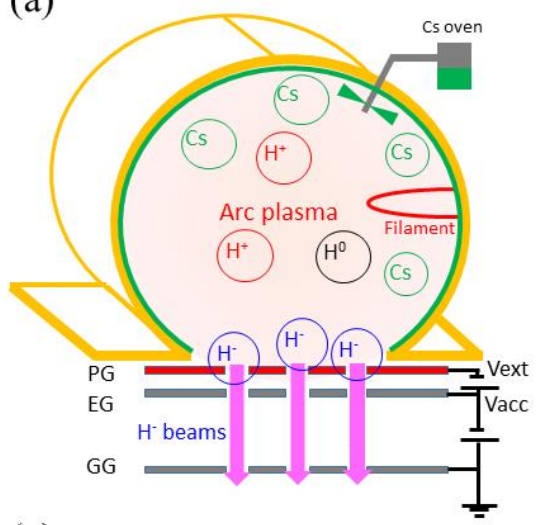

(c)

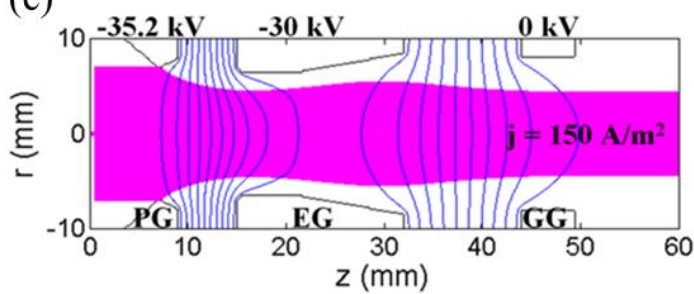

(b)

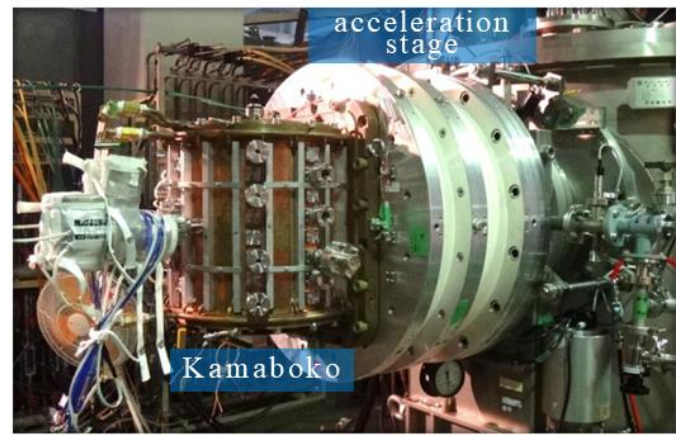

(d)

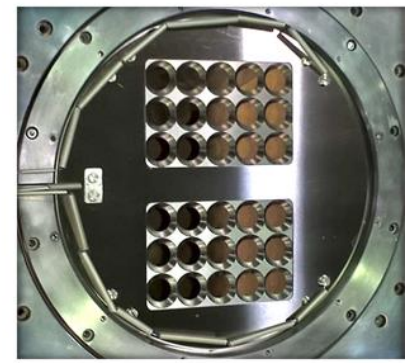

Figure 1. (a) sketch of NITS Kamaboko-type plasma source (b) picture of the Kamaboko installed on the NITS accelerator (c) example of a 2D electrostatic simulation of NITS accelerator with ITER-like EG geometry, using the SLACCAD code [13] (d) picture of the Plasma Grid showing two groups of 15 chamfered apertures, with a diameter of $14 \mathrm{~mm}$

Even though the ratio of extracted electron per negative ion is kept low by the filter field (around 1 in good conditions), the electrons that pass through the PG must be suppressed, otherwise the grid system would be damaged. For this reason, a magnetic field perpendicular to the beam propagation and to the Filter Field directions is generated by permanent magnets embedded in the EG (the Co-extracted Electron Suppression Magnets or CESM), so that almost all the co-extracted electrons are deflected and impinge on the EG. By convention on the coordinate system, the Filter Field will follow the horizontal direction, and the Electron Suppression Field the vertical direction.

Since the Electron Suppression Field orientation is alternate row by row, the suppression field also deflects the negative ions and causes the so-called "criss-cross" deflection of the beamlets that increases the divergence of the whole beam [5]. The beam deflection must be corrected to optimize the NBI efficiency, and the solution chosen in the case of NITS is to superpose to the CESM a second array of permanent magnets based on the principle of the Halbach array (Asymmetric Deflection Compensation Magnets or ADCM [4]). This solution has successfully corrected the criss-cross deflection of the beamlets during the second experimental campaign [2]. In NITS, this magnetic configuration was adopted for one half of the EG while the other half was kept with the basic configuration to allow a comparison. During the two campaigns, five magnetic configurations have been tested (Figure 2).

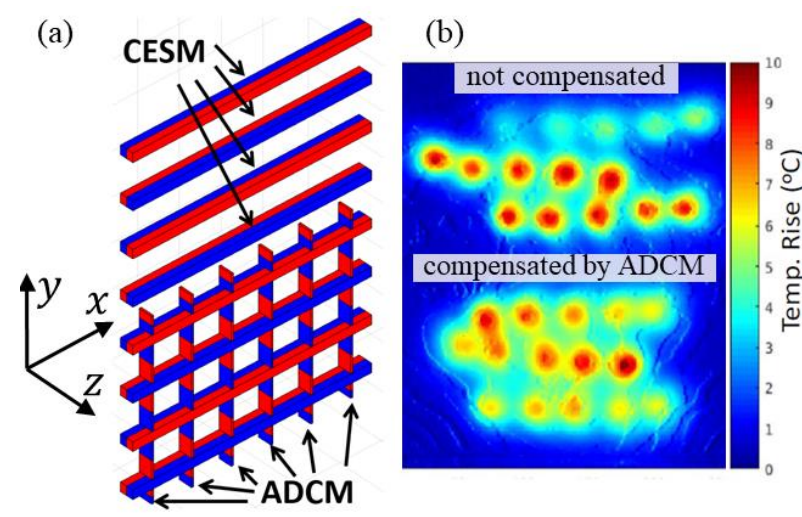

Figure 2. (a) layout of the permanent magnets embedded in the EG and frame of reference ( $\mathrm{z}$ is the beamlet direction) (b) example of thermal image on the CFC target showing the magnetic field effect on beamlet deflection

Table 1. magnetic configurations during experiments $\left(B_{r}\right.$ refers to the remanence of the permanent magnets)

\begin{tabular}{|c|c|c|}
\hline configuration type & B rCESM [T] & B rADCM [T] \\
\hline $1^{\text {st }}$ Joint Exp - phase 1 & 1.1 & 0.88 or 0 \\
\hline $1^{\text {st }}$ Joint Exp. - phase 2 & 1.1 & 1.1 or 0 \\
\hline $2^{\text {nd }}$ Joint Experiments & 0.77 & 1.1 or 0 \\
\hline
\end{tabular}




\subsection{Measurement of deflection}

About one meter after the acceleration stage exit, the beamlets deposit their power on a Carbon Fibre Composite (CFC) tile and an IR camera records the thermal footprint (Figure 2). The thermal images are numerically processed so as to obtain a time-dependent map of the temperature increase during the pulse. This map is fitted by a sum of 30 Gaussians [14], so that it is possible to determine the center coordinates and measure the absolute deflection of each single beamlet, provided that the exact position and orientation of the CFC tile relative to the GG is known. Since the horizontal deflection produced by the CESM is alternate row by row, we can assume that the average horizontal deflection of the beamlets is zero. This hypothesis formally implies that the deflection is an odd function of the vertical component of the suppression field, and this will be discussed when introducing the asymmetry of the current profile at the meniscus.

We call $\Delta x_{i}$ the horizontal displacement of the beamlet footprint on the CFC tile $(\Delta x>0$ for a beamlet deflected to the left). We consider two beamlets of a same column alternately deflected, their horizontal coordinates are given by:

$$
\left\{\begin{array}{c}
x_{\text {left }}=x_{\text {offset }}+\Delta x_{1} \\
x_{\text {right }}=x_{\text {offset }}-\Delta x_{2}
\end{array}\right.
$$

The average deflection induced by the suppression field is given by $\frac{1}{2}\left(x_{\text {left }}-x_{\text {right }}\right)$, and knowing the position of the CFC tile relatively to the grids is not necessary. In what follows, the deflection will be defined from the beam footprint by:

$$
\Delta x=\frac{1}{N_{\text {left }}} \sum_{i=1}^{\text {Nleft }} x_{i}-\frac{1}{N_{\text {right }}} \sum_{j=1}^{\text {Nright }} x_{j}
$$

where $\left\{x_{i}\right\}$ are the horizontal coordinates of the beamlet centers on the CFC target.

In this study, the deflection is described by a distance on the tile in order to stay close to the experimental data. It is a very reasonable hypothesis to assume that a beamlet exits the accelerator with a linear trajectory and a small angle, therefore the horizontal displacement and the angular deflection are directly proportional.

\section{Interpretation of the experiments and comparison with simulations}

\subsection{Simulations of the accelerator with Opera $3 d$}

To simulate the beam trajectory, different codes have been used with converging results. In this study, we will focus on the results with Opera Simulation Software [3]. Opera is a multiphysics FEA software that includes a module dedicated to the transport of charged particles (SCALA). It calculates in an iterative way the trajectory of a bunch of macro-particles (clusters of particles whose masses and charges are summed) by solving Poisson equation, taking account of the space charges and assuming a continuous beam and static fields. SCALA includes a set of models to describe the emission of particles from a surface. One model (Plasma Free Surface - type 103) simulates the extraction of charged particles from a plasma. The macro-particles start from a plane with a thermal velocity defined by the user, and they drift toward the extraction area without influence of the external fields until the meniscus, whose shape and position are determined in an iterative way to match the input parameters. The voltage and current density are defined by the user and constant over the meniscus surface.

The model of the NITS accelerator comprises a single aperture of each grid and a single beamlet. The $z$ axis is perpendicular to the grids and the suppression field follows the $y$ direction. The PG aperture center is at the coordinate $(x=0, y=0, z=0)$.

In the simulations, the horizontal angular deflection at a cross-section of the beam is defined as the mean value of the horizontal angular distribution of the beam, that is: $\theta(z)=\frac{\sum_{\text {macroparticules }} i_{n} \theta_{n}(z)}{\sum_{\text {macroparticules }} i_{n}}$ with $i_{n}$ and $\theta_{n}$ the current and horizontal deflection of the $n^{\text {th }}$ macroparticle.

$$
\theta_{n}(z)=\operatorname{Atan}\left(\frac{d x}{d z}\right)_{n}=\operatorname{Atan}\left(\frac{v_{x}(z)}{v_{z}(z)}\right)_{n}
$$

The angular deflection is computed after the exit of the accelerator, at the end of the simulation domain at a position $z_{\text {exit }}$. At this point the beam trajectory is considered linear, therefore the horizontal displacement on the CFC tile is given by:

$$
\begin{aligned}
\Delta x=x\left(z=z_{\text {exit }}\right) & +\left(z_{C F C}-z_{\text {exit }}\right) \\
& * \tan \left(\theta\left(z=z_{\text {exit }}\right)\right)
\end{aligned}
$$

In all the simulations presented here, the voltage ratio and perveance are kept constant and close to their optimum value (to minimize the beamlet divergence). This is also the case in most of the experiments considered.

\subsection{Results of the first simulations, comparison with the experiments}

As detailed in [2], the results of the first Opera model are very well fitted by a semi-analytical model (the expression is found analytically and the coefficients values comes from simulations): 


$$
\Delta x=\frac{A_{1} * B r_{C E S M}-A_{2} * B r_{A D C M}}{\sqrt{V_{\text {ext }}}}
$$

where $B r_{C E S M}$ (resp. $B r_{A D C M}$ ) is the remanence of the CESM (resp. ADCM), $A_{1}$ and $A_{2}$ are positive constants. The horizontal deflection decreases when increasing the ADCM remanence and vanishes at the design point. During the first campaign, the experimental deflection was in average $7 \mathrm{~mm}$ higher than predicted [5]. The fit (1) applied to these results indicates that the measured deflection should vanish for $B r_{A D C M}=1.1 T$ and $B r_{C E S M}=0.66 T$ providing that the offset does not vary with the magnetic configuration.

During the second campaign, the deflection was instead suppressed for $B r_{A D C M}=1.1 T$ and $B r_{C E S M}=$ $0.77 T$ [2][14]. A deviation with respect to the simulated deflection is still present, but lower than measured in the first campaign.

Two conclusions are drawn from those experimental campaigns:

- Under various experimental conditions a deviation is observed between the experiments and the simulation results;

- This deviation is larger when the vertical component of the magnetic field in the extraction area is higher.

The first point implies that at least one of the hypotheses of the Opera model is wrong. Following previous work on this topic we assume that the current distribution at the meniscus is not radially-symmetric. We suppose that this asymmetry arises from a drift of the plasma in the source due to the magnetic field [9], and that this effect is thus larger for higher magnitude of the magnetic field inside the source (following the second point).

\section{Beam model with an asymmetric current density at the meniscus}

\subsection{Description of the model}

In Opera 19, a model of emitter (User Defined Emitter - type 9) can reproduce the Free Plasma Surface emitter with a custom current density profile over the meniscus. This emitter is used in a new model that includes current asymmetry of the beamlet. The conventions about the origin and orientation of the coordinate system are the same. If the electronsuppression magnetic field is directed toward $-\boldsymbol{y}$ inside the source, then the negative ions drift towards $-\boldsymbol{x}$ and the current density is higher on this side of the meniscus. To perform a systematic study of the asymmetry effect, the unbalancing of the current density at the meniscus must be quantified by a figure of merit. The following hypotheses are made in the model:

i) $\quad j_{\text {left }}$ and $j_{\text {right }}$ are the average current densities of both halves of the meniscus and $j_{\text {tot }}=\frac{1}{2}\left(j_{\text {left }}+j_{\text {right }}\right)$ corresponds to the current density measured by calorimetry in the experiments

ii) We define the unbalancing factor as $\alpha_{\text {unb }}=\frac{j_{\text {left }}-j_{\text {right }}}{j_{\text {left }}+j_{\text {right }}}$

iii) We assume that the absolute deviation to the average current density $\left|j(x, y)-j_{\text {tot }}\right|$ is symmetric to $x=0$ (centered profile)

iv) We assume that the unbalancing factor is proportional to the vertical component of the magnetic field at the meniscus and we define the reduced unbalancing factor $\widetilde{\alpha_{u n b}}=\frac{\alpha_{\text {unb }}}{\text { By,meniscus }}$. With this assumption it is correct to measure the deflection as described in . since the additional deflection is still an odd function of the magnetic field vertical component.

These four conditions are not enough to discern a specific current density profile at the meniscus. To compare with simplicity the beamlet deflection and the unbalancing factor, a stepwise current density profile has been chosen (Figure 5). It is necessary to demonstrate that this choice has no large impact on the beam optics, and that the discontinuity at the profile center generates no physical inconsistency. To do so we compared the optical properties of the beamlet (without magnetic field) for three different current density profiles respecting the hypotheses defined above. The results are shown in Table 2 and evidence no significant variations of divergence and deflection.

Table 2. Beamlet optics at the end of the simulation domain for different profiles of current density at meniscus with $\alpha_{u n b}=0.2$ and $j_{\text {tot }}=140 \mathrm{~A} \cdot \mathrm{m}^{-2}$

\begin{tabular}{|c|c|c|c|}
\hline profile & 1: stepwise & 2: linear & 3: smooth step \\
\hline$\langle x\rangle[m m]$ & 0.41 & 0.45 & 0.43 \\
\hline horizontal deflection $[$ mrad $]$ & 8.15 & 8.95 & 8.70 \\
\hline e-folded divergence $[\mathrm{mrad}]$ & 15.32 & 15.53 & 15.37 \\
\hline
\end{tabular}




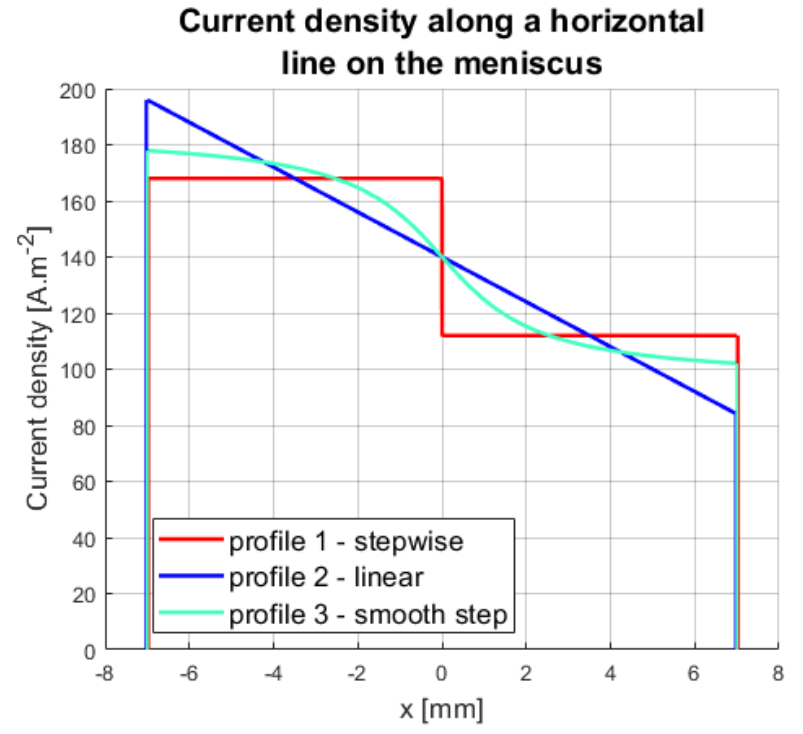

Figure 3. Representation of different tested current density profiles at meniscus, with $\mathrm{R}_{\mathrm{PG}}=7 \mathrm{~mm}, \mathrm{~V}_{\text {ext }}=4.4 \mathrm{kV}$, j, $\mathrm{j}_{\text {tot }}=$ $140 \mathrm{~A} \cdot \mathrm{m}^{-2}$ and $\alpha_{\mathrm{unb}}=0.2$ and varying steepness for the connection between the left and right parts

It is thus reasonable to draw general conclusions about the profiles respecting the four hypotheses previously defined from the study of a simple stepwise profile. More details about Opera models with asymmetric, stepwise current density profile can be found in [2] and [14].

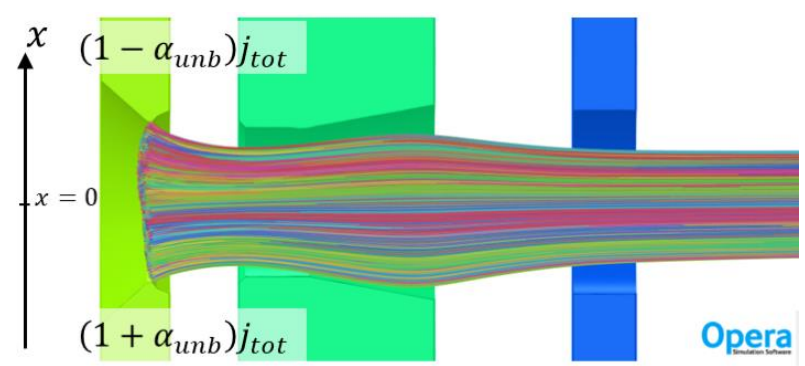

Figure 4. Opera simulation of a beamlet with stepwise asymmetric current density at meniscus

\subsection{Changes with respect to a radial symmetric case}

In a case without magnetic field, the asymmetric beamlet is deflected toward the direction of lower current density at the meniscus (here $x>0$ ), and the deflection evolves linearly with the unbalancing factor, as shown in Figure 5.

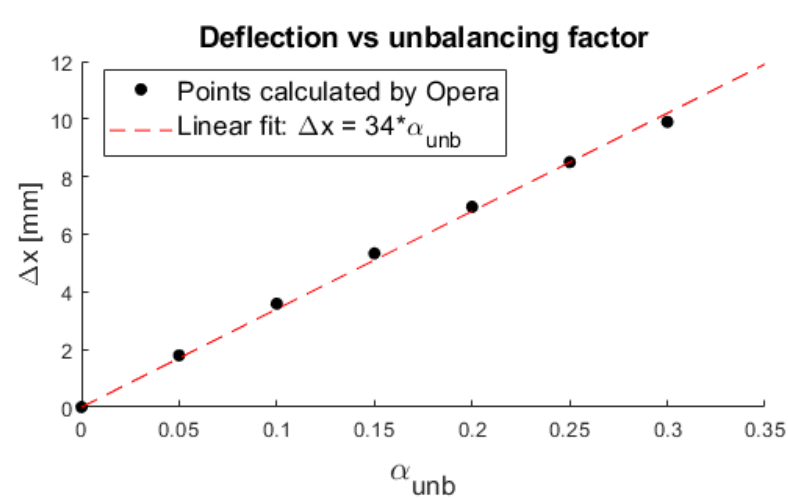

Figure 5. With invariant operating parameters, the deflection is linear with the unbalancing factor

To get a clearer idea of the effect of the asymmetry, the beamlet trajectory was studied for different values of $\alpha_{u n b}$. In Figure 6 the average position of the beam and the evolution of the unbalancing factor at a beam section $\alpha_{u n b, z}$ (applying the definition of 3.1. to a crosssection of the beamlet at position $z$ ) are plotted along the beam axis for different values of $\alpha_{u n b}$ at the meniscus.

We see in the simulations (Figure 6) that $\alpha_{u n b, z}$ and $\langle x\rangle(z)$ are proportional to the unbalancing factor at the meniscus $\alpha_{u n b}$. We present here a short description of different effects of the unbalancing on the deflection, inspired by [8].

i) The part of the beam with higher current density has a higher weight in the averaged quantities. Consequently, the initial average position and deflection of the beam are not zero but are proportional to the unbalancing factor. This is visible in Figure 6.

ii) Because of the asymmetry, the average space charge force on the macro-particles is not initially zero. The self-electric field magnitude is stronger in the half of the beamlet with higher current density, which results in a global electric force directed toward the opposite half. The self-electric force induces a deflection of the ions that tends to balance the current distribution until $\alpha_{u n b, z}$ vanishes (Figure 6).

iii) The beam reaches the PG with an offset proportional to $\alpha_{\text {unb }}$ towards the part with higher current density, and is deflected in the opposite direction by the electrostatic lens (Davisson-Calbick lens effect [15]). The deflection induced is proportional to the offset of the beamlet at the EG and therefore to the unbalancing factor.

Those three effects contribute to the final deflection and each of them is linear with $\alpha_{u n b}$. 

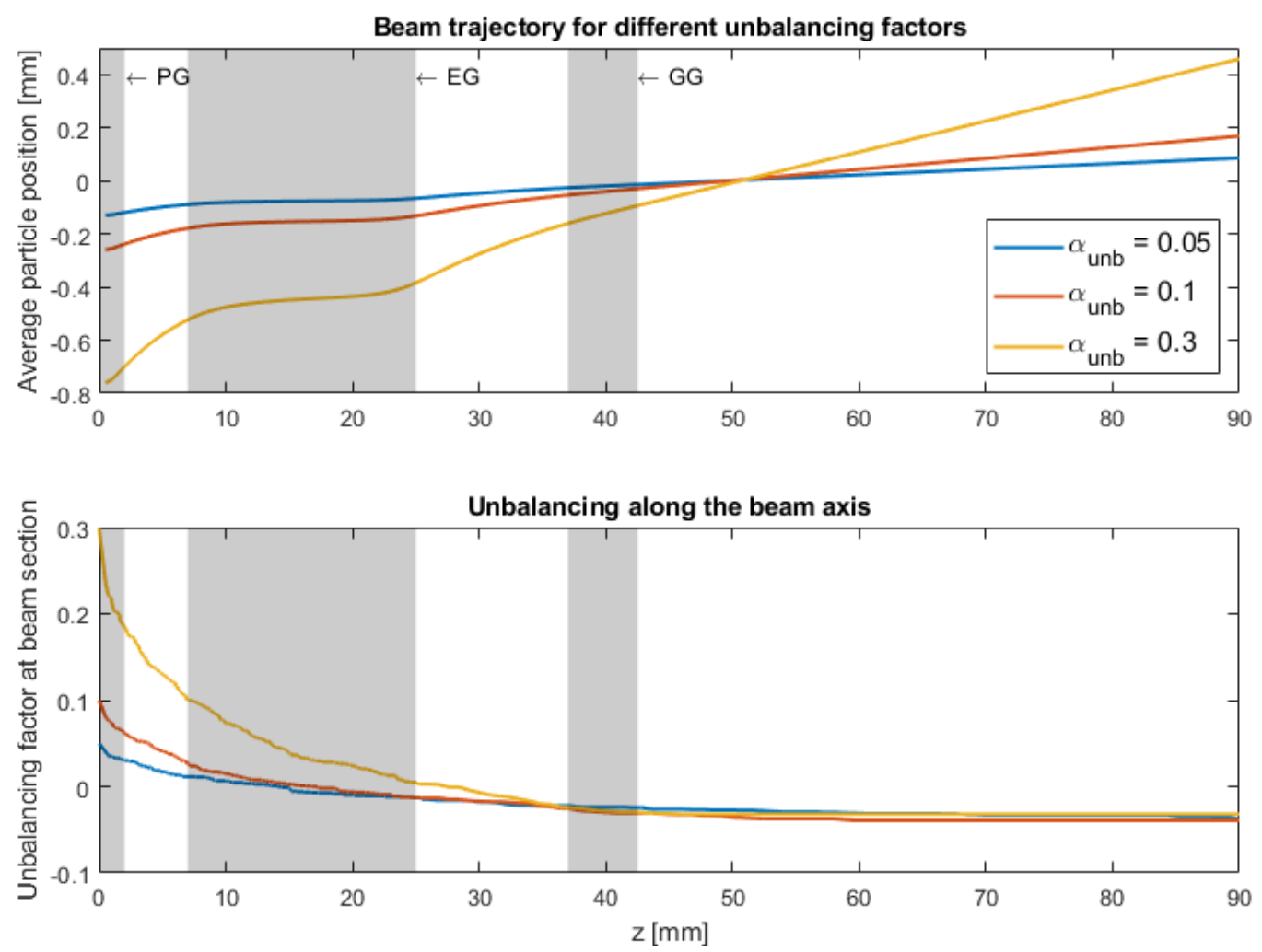

Figure 6. The trajectory of the beam is computed without magnetic field (in a symmetric case the trajectory would follow the $x=0$ axis). The particle trajectories are modified in a way that tends to cancel the asymmetry. The beamlet enters the EG with an offset proportional to the unbalancing factor and is deflected by the electrostatic lens, the final deflection is proportional to $\alpha_{u n b}$

The deflection is very sensitive to the unbalancing factor and a small unbalancing (around 0.2) is enough to explain the difference measured during the Joint Experiments. The final deflection obtained by Opera simulation is well fitted by modifying the equation (1):

$$
\Delta x=\frac{A_{1} * B r_{C E S M}-A_{2} * B r_{A D C M}}{\sqrt{V_{\text {ext }}}}+A_{3} * \alpha_{u n b}
$$

In Table 3, the deflection calculated by (2) is compared to the results of Opera simulations for various sets of parameters: the root-mean-square error (RMSE) is small and the maximum deviation is less than $1 \mathrm{~mm}$. This means that, in the rest of the study, the formula (2) can be used to predict the results of the simulations with Opera. This makes the comparison between Opera simulations and the experiments much easier as it is not necessary to simulate each single case, instead the deflection predicted by (2) with the constants of Table 3 is compared to the measurements.

Table 3. Comparison between the formula (2) and the Opera simulations for various sets of parameters

\begin{tabular}{|c|c|c|c|c|}
\hline$A_{1_{\text {opera }}}$ & $A_{2_{\text {opera }}}$ & $A_{3_{\text {opera }}}$ & Data & RMSE \\
\hline $22.8 \mathrm{~mm} \cdot \mathrm{kV}^{1 / 2} \cdot \mathrm{T}^{-1}$ & $24.6 \mathrm{~mm} \cdot \mathrm{kV}^{1 / 2} \cdot \mathrm{T}^{-1}$ & $32.1 \mathrm{~mm}$ & $\begin{array}{l}\text { - } 90 \text { simulations } \\
\text { - } \quad V_{\text {ext }} \text { from } 2.5 \mathrm{kV} \text { to } 8.5 \mathrm{kV} \\
\text { - } \quad B r_{C E S M} \text { from } 0.55 \mathrm{~T} \text { to } 1.1 \mathrm{~T} \\
\text { - } \quad B r_{A D C M} \text { from } 0 \text { to } 1.1 \mathrm{~T}\end{array}$ & $0.53 \mathrm{~mm}$ \\
\hline
\end{tabular}

\subsection{Application to the Joint Experiments}

This simplified model is now applied to the analysis of the Joint Experiments results, by finding for each case the value of $\alpha_{u n b}$ necessary to match $\Delta x$ in the experiments and the simulations. As shown in Figure 7, a first simplified analysis of the experimental data shows a roughly linear evolution of $\alpha_{\text {unb }}$ with the vertical component of the magnetic field at the meniscus for a given value of the arc power, as suggested in 3.1. 


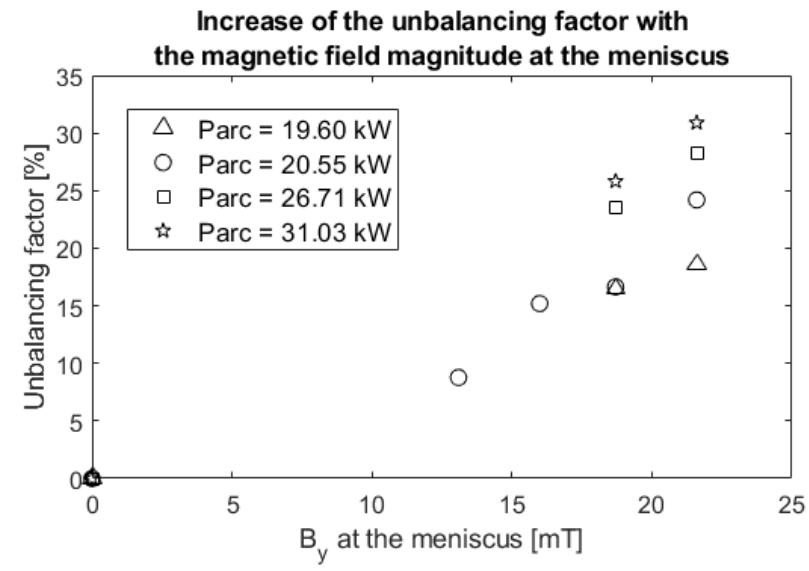

Thus, we assume $\alpha_{u n b}=\widetilde{\alpha_{u n b}} B_{y, \text { meniscus }}$ with $\widetilde{\alpha_{u n b}}$ independent on the magnetic field, and we can now use equation (2) to deduce the value of $\widetilde{\alpha_{u n b}}$ to be put in the simulations to match the experimental value of deflection $\Delta x_{\text {exp }}$.

Figure 7. Asymmetry coefficient $\alpha_{\text {unb }}$ obtained from a simplified analysis of the Joint Experiments

$$
\widetilde{\alpha_{\text {unb }}}=\frac{1}{A_{3} * B_{y, \text { meniscus }}}\left(\Delta x_{\text {exp }}-\frac{A_{1} * B r_{C E S M}-A_{2} * B r_{A D C M}}{\sqrt{V_{\text {ext }}}}\right)
$$

At this point, the asymmetry of the current distribution is still an ad hoc hypothesis and the value of $\widetilde{\alpha_{u n b}}$ is determined only by the requirement to conciliate the measurements and the simulations. The unbalancing of the current density at the meniscus can explain the difference observed, but has no predictive ability as the unbalancing factor is deduced ex post from the measurements, with a different value for each set of operating parameters.

To go further we try to link the values of $\widetilde{\alpha_{u n b}}$ with the operating parameters. If we compare $\widetilde{\alpha_{u n b}}$ with the arc power of the source $P_{\text {arc }}$ (the electrical power injected through the tungsten filament) for the impulses with the best optics (small co-extraction ratio and a small divergence), we observe a correlation (Figure 8).
The Pearson coefficient of correlation between $P_{\text {arc }}$ and $\widetilde{\alpha_{u n b}}$ for 357 cases with various operating parameters is 0.43 . We interpret this value as a weakmoderate linear relation and we use it to define $\widetilde{\alpha_{u n b}}$ independently from the Opera simulations as the reduced unbalancing factor of the real beamlet, estimated by $A_{4} * P_{\text {arc }}$ where $A_{4}$ is an empirical constant supposed to be independent on the operating parameters.

The value of the unbalancing factor to use in Opera simulations is no longer chosen to fit the experimental results but is given by $\alpha_{u n b}=A_{4} * P_{\text {arc }} * B_{y \text {,meniscus }}$. The deflection as a function of the operating parameters is estimated independently from the Opera simulations by the semi-empirical formula (3):

$$
\Delta x=\frac{A_{1} * B r_{C E S M}-A_{2} * B r_{A D C M}}{\sqrt{V_{\text {ext }}}}+A_{3} * A_{4} * B_{y, \text { meniscus }} * P_{\text {arc }}
$$

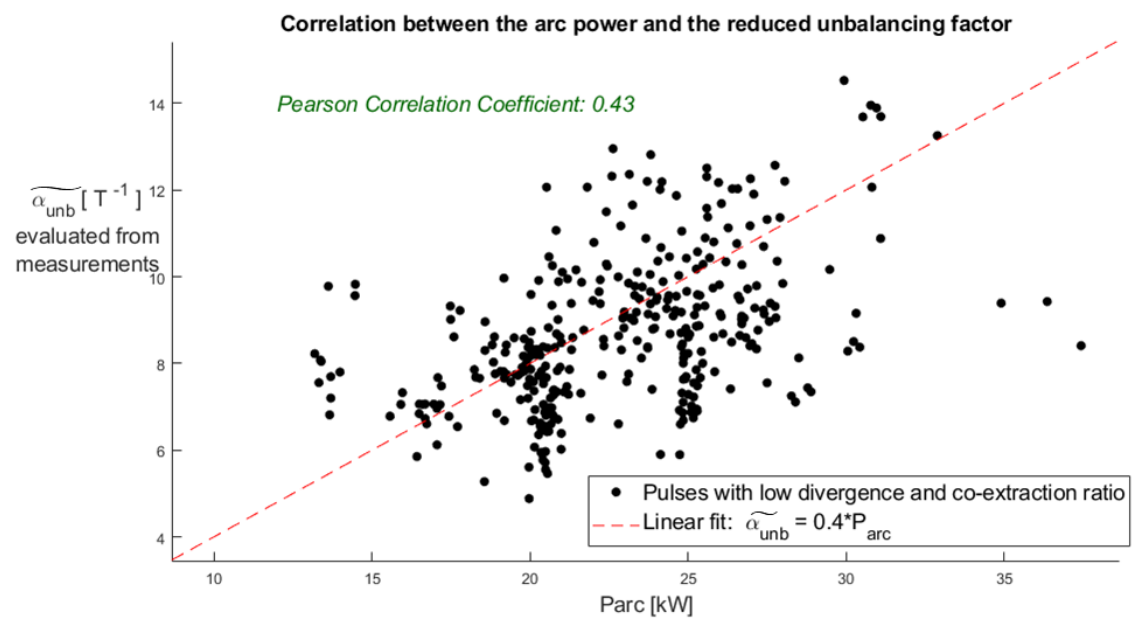

Figure 8. Correlation between the reduced unbalancing factor $\widetilde{\alpha_{u n b}}$ and the applied arc power Parc. A weak-moderate linear relation is observed between those parameters 
In Table 4, Figure 9 and Figure 10 the measurements of deflection are compared to the results of Opera simulations, first without unbalancing, and then with a reduced unbalancing factor $\widetilde{\alpha_{u n b}}=A_{4} * P_{\text {arc }}$. A large deviation is observed in the first case (as explained in 1) while the second case is much more accurate. This confirms that assuming a non-radial symmetric current density distribution at the meniscus allows to conciliate the simulations with the measurements, and to predict the deflection of the beamlet.

Table 4. Comparison between the formula (3), the Opera simulations and the experimental results

\begin{tabular}{|c|c|c|c|c|c|c|}
\hline & $A_{1_{\text {exp }}}$ & $A_{2} \exp$ & $A_{3_{\text {exp }}}$ & $A_{4} \exp \left[k W^{-1} \cdot T^{-1}\right]$ & Data & RMSE \\
\hline $\begin{array}{l}\text { Opera simulations (coefficients of } \\
\text { Table 3) with } \alpha_{u n b}=0\end{array}$ & 22.8 & 24.6 & I & I & $\begin{array}{l}752 \text { measurements } \\
V_{\text {ext }} \text { from } 0.75 \text { to } 5.6 \mathrm{kV}\end{array}$ & $6.19 \mathrm{~mm}$ \\
\hline $\begin{array}{l}\text { Opera simulations (coefficients of } \\
\text { Table 3) assuming } \widetilde{\alpha_{u n b}}\left[T^{-1}\right]= \\
0.4 P_{\text {arc }}[k W](\text { Figure 8) }\end{array}$ & 22.8 & 24.6 & 32.1 & 0.4 & $\begin{array}{l}P_{\text {arc }} \text { from } 13.2 \text { to } 37.4 \mathrm{~kW} \\
\alpha_{u n b} \text { from } 9.9 \text { to } 32.4 \%\end{array}$ & $1.89 \mathrm{~mm}$ \\
\hline
\end{tabular}

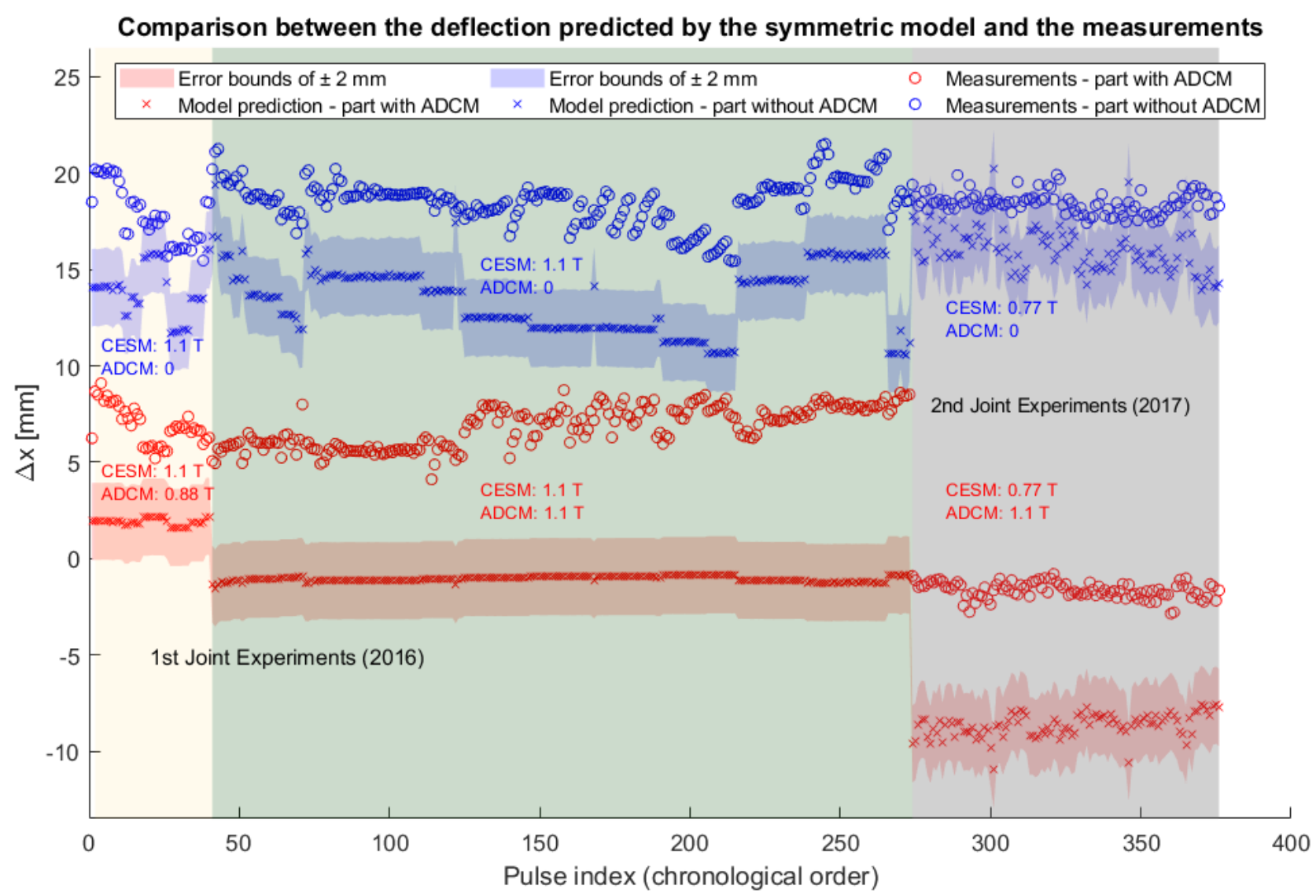

Figure 9. The deflection simulated by Opera with a radial symmetric meniscus is compared to 752 measurements with various operating parameters and five magnetic configurations. $94 \%$ of the measurements are outside of the model predictions with an error bound of $\pm 2 \mathrm{~mm}$. 


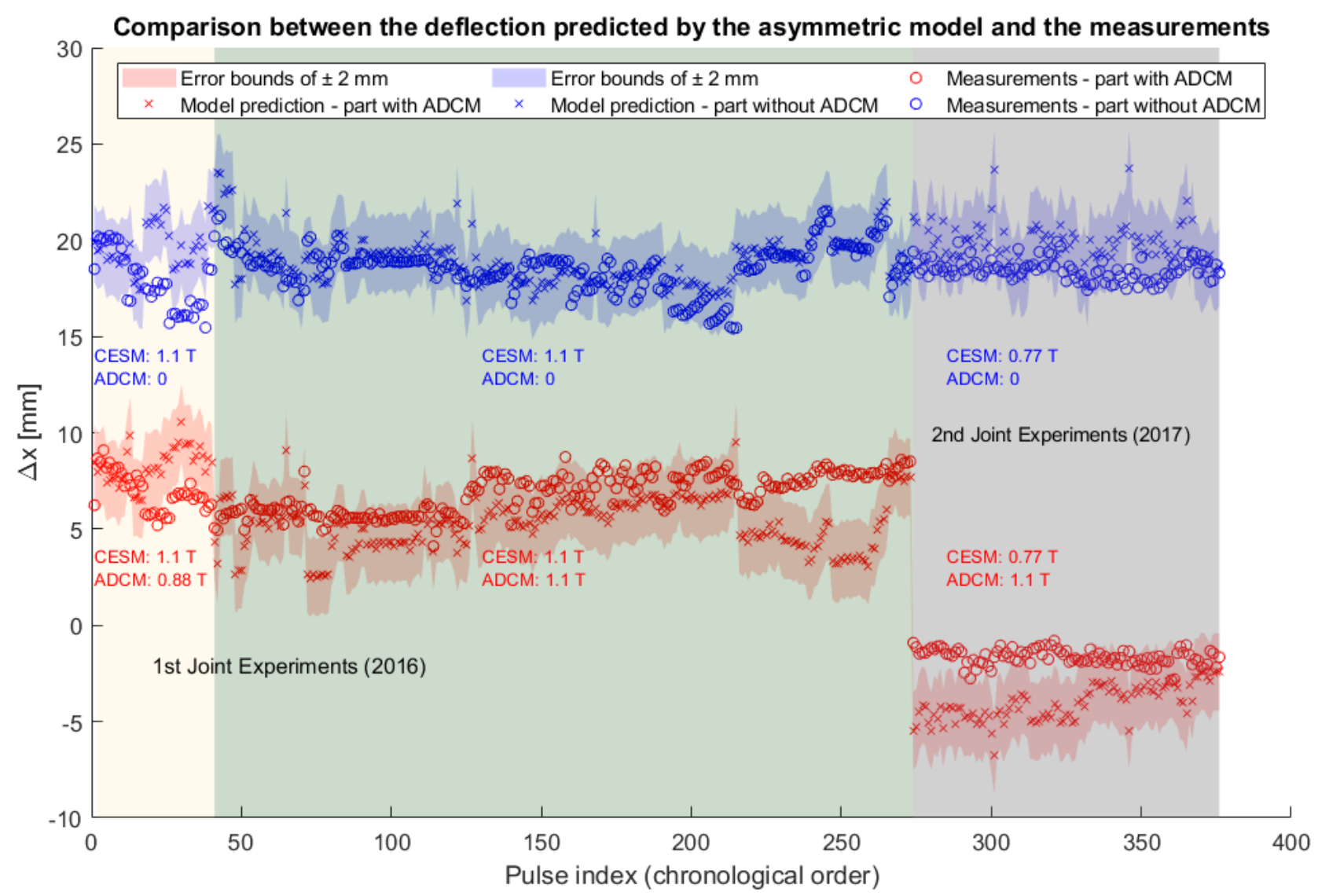

Figure 10. The 752 measurements are now compared to the results of Opera when a non-radial symmetric profile is considered, with a value $\alpha_{u n b}$ proportional to the source arc power and to the magnetic field vertical component at the meniscus. The model reproduces $70 \%$ of the results with an error bound of $\pm 2 \mathrm{~mm}$

The simulations of the acceleration stage and the analyses of the Joint Experiments have led to the following conclusions:

(i) In the case of NITS accelerator, it is possible to conciliate the Opera simulations with the experimental results for various sets of operating parameters and magnetic configurations by rejecting the assumption of a radial symmetric current density at the meniscus.

(ii) The asymmetry of the current density profile is described by a figure of merit $\alpha_{\text {unb }}$ that is roughly proportional to the vertical component of the magnetic field at the meniscus and to the arc power of the source filaments.

In the last part of this paper, those conclusions are investigated by a simulation of the plasma source.

\section{Simulations of the ion source}

\subsection{Description of the code}

In this part, we present the results of a simulation of a plasma source and extractor with a 3D particle-in-cell Monte Carlo collisions (PIC-MCCs) explicit algorithm. The code is parallelized in a hybrid manner using OpenMP [16] and MPI libraries. From the extractor geometry and plasma parameters, the code computes the trajectories and densities of the plasma species and the electrostatic field in the simulation domain.

The filter field is approximated by a Gaussian profile along the axis, and the suppression field by a cusp field surrounding the PG aperture. Both fields are located in perpendicular planes defined as $(x, z)$ (filter) and $(y, z)$ (suppression). The suppression field is directed along-y and the beamlet propagates from $-z$ to $z$.

To reduce the computation time, the plasma is scaled down in density by scaling the vacuum permittivity $\widetilde{\varepsilon_{0}}=64 \varepsilon_{0}$ and the extractor dimensions are reduced (the PG has a radius of $4 \mathrm{~mm}$, a thickness of $2 \mathrm{~mm}$ and the extraction gap is $3.5 \mathrm{~mm}$ long). A single circular aperture is modelled with periodic boundary conditions, and the geometry of the grid is approximated by ignoring the chamfers. The cell size is $125 \mu \mathrm{m} \times 125 \mu \mathrm{m} \times 125 \mu \mathrm{m}$, which is smaller than the scaled Debye length $\left(\Delta_{\text {cell }} / \lambda_{D}=0.814\right)$. The extraction voltage is $400 \mathrm{~V}$ and the perveance is close to the optimum value. The magnetic field vertical component at the meniscus is around $0.02 T$, which is quite similar to the values for NITS.

A detailed description of the numerical constraints, the parallelization, the scaling, the physical phenomena included and the magnetic field shape is found in [9].

\subsection{Emergence and quantification of the current density asymmetry in the source}


Since the electrons are magnetized in the extraction area, the filter and suppression magnetic fields generates a transverse electronic transport. The presence of the walls induces a charge separation and a polarization drift of the negative ions upstream of the meniscus and at the entrance of the PG, as shown in Figure 11. A higher flux of particles is directed towards one side of the aperture, which results in a non-radial symmetric current distribution inside the aperture. This is visible for example in Figure 11 a), where the flux of negative ions is higher on the bottom part $(x<0)$ of the PG aperture. A more advanced description of the plasma parameters asymmetry caused by the plasma drift is found in [9] and [10].
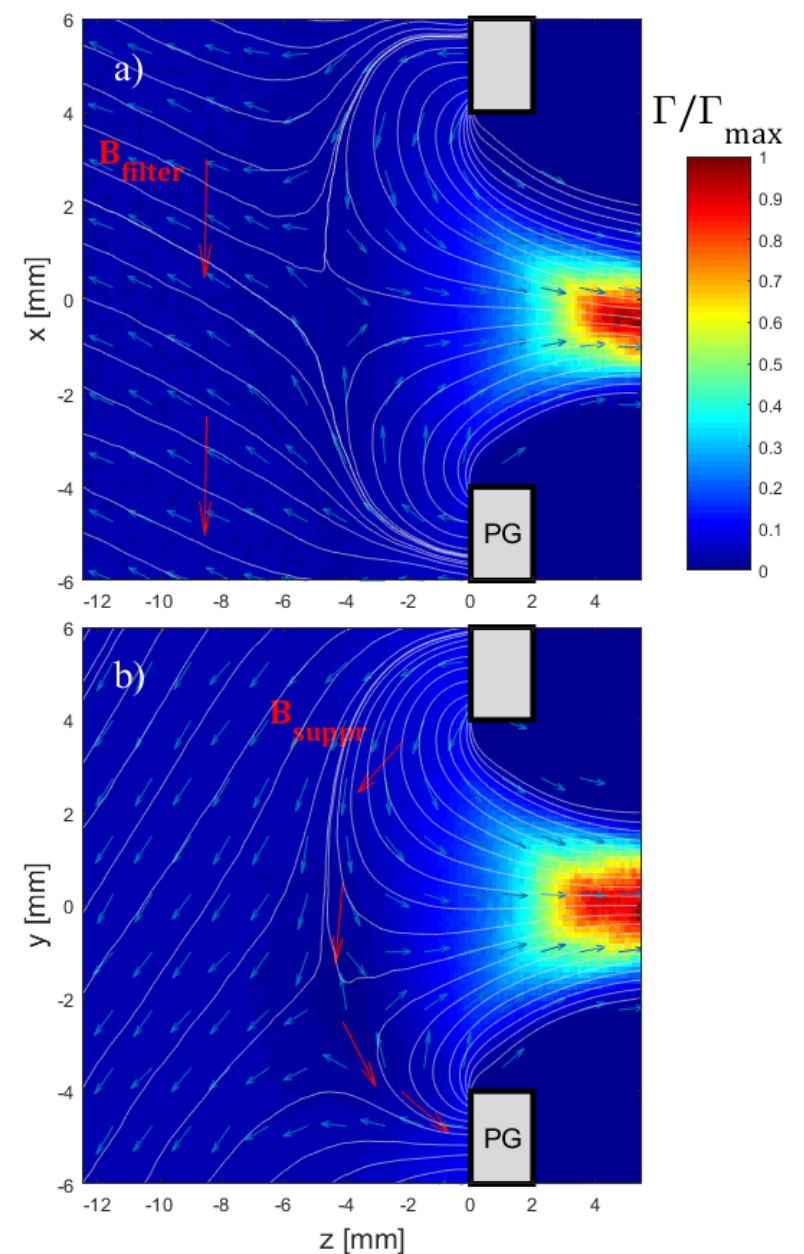

Figure 11. Flux of $\mathrm{H}^{-}$ions in the source (left) and the acceleration gap (right) in the planes (x,z) (a) and (y,z) (b). The white line and blue vectors represent the flux streamlines, the red vectors represent the magnetic field in the plane

The simulation provides current density maps in the planes $(x, z)$ and $(y, z)$. The unbalancing factor $\alpha_{u n b}$ (3.1). that was heuristically evaluated from measurements in the previous parts of this paper is now explicitly computed.

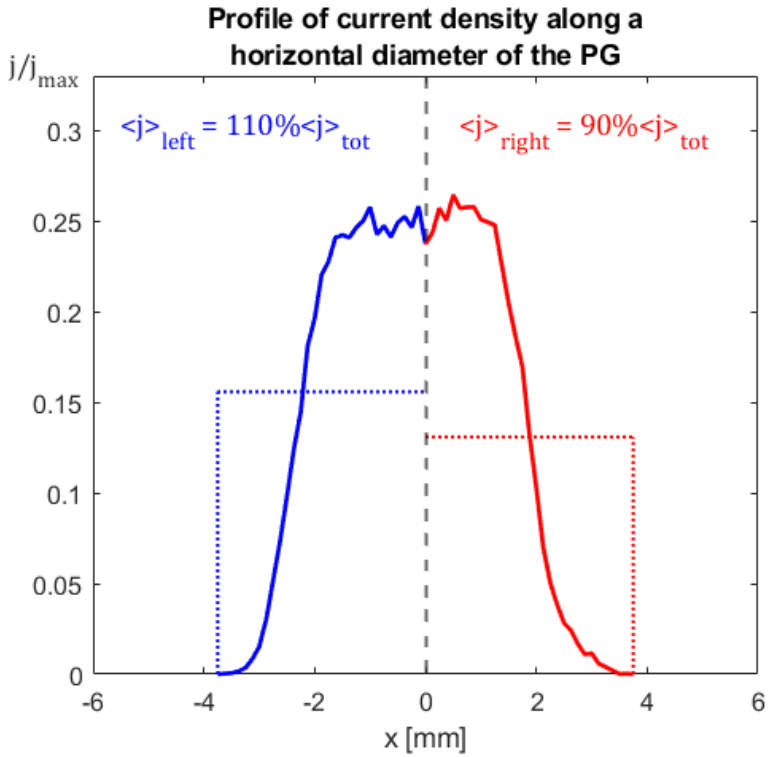

Figure 12. Current density profile along $x$ in the middle of the PG. The unbalancing factor at this point is $10 \%$. The dashed line corresponds to the middle point of the current distribution. The dotted line represents the stepwise profile that would be used in Opera to approximate the current density profile.

Inside the PG the average value of $\alpha_{u n b}$ is $9.1 \%$, which is lower than assumed in the experiments with NITS (see Table 4). This difference is expectable since the plasma parameters are scaled down and the accelerator size reduced in the PIC simulation.

Because of the filter field, a vertical asymmetry is also present in the beamlet, but much weaker than the horizontal one, as can be seen by eye in Figure 11. The average value of the vertical unbalancing inside the PG is $1.3 \%$.

\subsection{Issues with the quantification of the asymmetry and further steps}

To apply the definition of $\alpha_{u n b}$ given in 3.1, it is necessary to define the "right half" and "left half" of the beamlet. This distinction is actually delicate in the frame of PIC simulations, because the vertical centerline that separates the beam envelope in two semicircles of same area is not defined as clearly as it is with ray-tracing codes, and a small error on the centerline position results in a significant error on $\alpha_{u n b}$.

Furthermore, the current density profiles (for example plotted in Figure 12) are not centered profiles like those used in Opera (plotted in Figure 3), which disproves the hypothesis made in 3.1.iii). In future steps, the current density should be approximated by a more realistic profile where the $\alpha_{u n b}$ quantity could be delicate to compute.

To avoid the difficulties encountered to set the value of $\alpha_{\text {unb }}$, a quantity that could be studied is the skewness ( $3^{\text {rd }}$ moment) of the particle horizontal position distribution.

$$
\mu_{2}=\left\langle(x-\langle x\rangle)^{2}\right\rangle
$$




$$
\mu_{3}=\frac{\left\langle(x-\langle x\rangle)^{3}\right\rangle}{\sigma^{3}}
$$

From the PIC simulation we get the position and velocity of the particles on the plane $(x, y)$ at the entrance of the EG, therefore we can compute the skewness of the distribution in this plane.

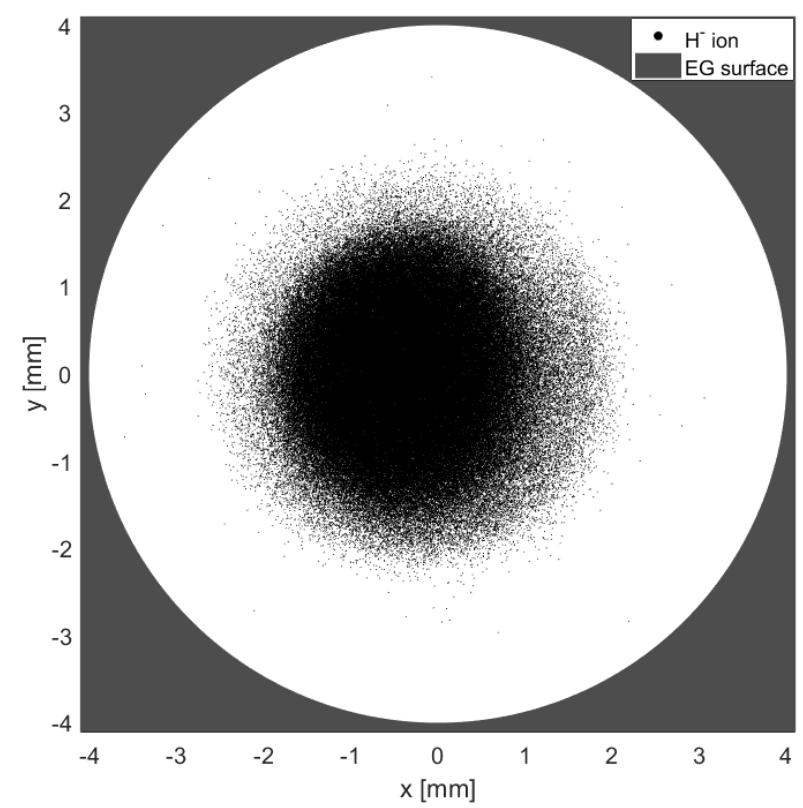

Figure 13. Cross-section of the beam at the EG. It is discernable by eye that there is a higher density of particles on the side $\mathrm{x}<0$, which is the direction toward which the beamlet is deflected in the extraction gap

The distribution of horizontal position of the particles has a variance $\mu_{2}=0.52 \mathrm{~mm}^{2}$ and a skewness $\mu_{3}=0.21$. Instead, a symmetric normal distribution with similar variance and sample size ( 300000 elements) has a skewness of \pm 0.043 with a $6 \sigma$-confidence level. The skewness computed from PIC simulation is not a simple statistic fluctuation but a clear characterization of the beamlet asymmetry. The third moment allows to quantify the asymmetry without assumptions about the current density profile and the beam envelope.

\section{Conclusions}

This work is a continuation of previous studies on the effect of a non-radial symmetric current density distribution of an $\mathrm{H}^{-}$ beamlet on its deflection in an accelerator [8][2]. It is confirmed by comparison of Opera models that the assumption of a radial-symmetric current density distribution made in most ray-tracing codes is too restrictive. Indeed, the beamlet deflection significantly increases even for a small asymmetry. We proposed a way to easily quantify the asymmetry (by a quantity $\alpha_{u n b}$ ) and implement it in raytracing simulation.
The asymmetry is proposed here as a physical explanation of the mismatch between the beamlet deflection calculated by beam optics codes and the experimentally measured deflection. The analysis of a large amount of experimental measurements obtained during two experimental campaigns allowed to heuristically evaluate the value of the unbalancing factor for each pulse and follow its evolution with the operational parameters.

This analysis of measurements (together with a physical understanding of the phenomenon causing the asymmetry) suggests us that the unbalancing factor is approximatively proportional to the magnetic field vertical component at the meniscus and the external power injected in the plasma. With these hypotheses, the beamlet deflection is predicted with a much better accuracy, with ray-tracing codes or by the simple formula (3) (Table 4, Figure 10).

The conclusions drawn from experimental data analysis are tested by a PIC-MCC simulation. We observe inside the PG a horizontal asymmetry in the same direction as assumed in Opera. $\alpha_{u n b}$ has a value of about 9\% (lower than the 10-30\% deduced from the experiments, which is expectable since the plasma density and the grids dimensions are scaled down).

The long-term goal of this approach is to perform accelerator simulations with input conditions precise enough to accurately predict the beamlet trajectory and general enough to do it with reasonable computing resources. Some points will be subject to future development:

- $\alpha_{u n b}$ is difficult to rigorously define in PIC simulations and has a simple geometric definition which makes it less physical than other possible characterizations of the asymmetry. Instead the asymmetry should be characterized for example by the skewness ( $3^{\text {rd }}$ moment) of the particle distribution, and a more realistic current density profile than a step function should be defined at the meniscus.

- The dependence of the asymmetry on the magnetic field magnitude and the injected power should be tested by PIC simulations to improve the predictive ability of the model.

\section{Acknowledgements}

This work was funded partially by Fusion for Energy under the Contract No. F4E- RFX PMS_A-WP-2015, 2016 and 2017. This publication reflects the views only of the authors, and Fusion for Energy cannot be held responsible for any use that may be made of the information contained therein.

\section{Author contributions}

S.D. developed the semi-analytical model and other numerical beam optics models and tools, and analysed the 
large amount of experimental data obtained during the Joint Experiments at QST. He wrote the first version of this paper.

A.D. developed and validated numerical models for beam optics and heat load simulations and applied them to the analysis of the Joint Experiments results.

G.F. developed 2D and 3D PIC models of a scaled down plasma source, used to study the current density asymmetry in a configuration similar to the kamaboko

F.T. and P.M. developed a simplified 3D PIC numerical simulation of the full kamaboko plasma source of NITS.

M.I. and J. H. organized and were responsible for the preparation, operation and scientific exploitation of the NITS accelerator during the Joint Experiments.

A.K, M.K. and G.C. conceived and promoted the Joint Experiments carried out on NITS with ADCM configuration in 2016 and 2017. G.C. also proposed the idea of using the results of NITS experiments in combination with PIC simulations for analysing the meniscus behaviour and validating the ad hoc hypothesis on the asymmetry of the current density. He also supervised the work related to this paper.

\section{References}

[1] H.P.L. de Esch et al,. Physics design of the HNB accelerator for ITER, 2015, Nucl. Fusion 55, 9, https://doi.org/10.1088/00295515/55/9/096001

[2] G. Chitarin et al., Improving a negative ion accelerator for next generation of neutral beam injectors: Results of QST-Consorzio RFX collaborative experiments, Fusion Engineering and Design, 146, Part A, 2019, Pages 792-795, ISSN 0920-3796, https://doi.org/10.1016/j.fusengdes.2019.01.081.

[3] https://operafea.com/

[4] G. Chitarin et al.: Cancellation of the ion deflection due to electron-suppression magnetic field in a negative-ion accelerator, Review of Scientific Instruments, 85, Issue 2, 02B317 (2014), doi: 10.1063/1.4826581

[5] G. Chitarin, et al., Experimental validation of an innovative deflection compensation method in a multi-beamlet negativeion accelerator, AIP Conference Proceedings. 1869, 030026 (2017); https://doi.org/10.1063/1.4995746

[6] N. Umeda, et al., Long-pulse beam acceleration of MeV-class Hion beams for ITER NB accelerator, Review of Scientific Instruments 85, 02B304 (2014); doi: 10.1063/1.4825161

[7] R. Gutser et al., Transport of negative hydrogen and deuterium ions in RF-driven ion sources, 2010 Plasma Phys. Control. Fusion 52 045017, doi: 10.1088/0741-3335/52/4/045017

[8] P. Veltri et al., Effects of Negative Ion Source Characteristics on Beam Optics: The Case of SPIDER, in IEEE Transactions on Plasma Science, 40, no. 9, pp. 2279-2284, Sept. 2012. doi: 10.1109/TPS.2012.2207742

[9] G. Fubiani, L. Garrigues, and J. P. Boeuf, Modeling of negative ion extraction from a magnetized plasma source: Derivation of scaling laws and description of the origins of aberrations in the ion beam, Phys. Plasmas 25, 023510 (2018); https://doi.org/10.1063/1.4999707

[10] F Taccogna and P Minelli, PIC modeling of negative ion sources for fusion, 2017 New J. Phys. 19 015012., https://doi.org/10.1088/1367-2630/aa5305

[11] P. Veltri et al., Ion beam transport: modelling and experimental measurements on a large negative ion source in view of the ITER heating neutral beam, 2017, Nucl. Fusion 57, 1, https://doi.org/10.1088/0029-5515/57/1/016025

[12] S. Abe et al., Effects of the extraction voltage applied by the puller-electrode on the $\mathrm{H}$ - extraction in the Linac4 negative ion source, AIP Conference Proceedings 1869, 030051 (2017), https://doi.org/10.1063/1.4995771

[13] D. Aprile et al., Realization of a Magnetically Compensated Extraction Grid for performance improvement of next generation NBI, Fusion Engineering and Design, 123, 2017, Pages 400-405, ISSN 0920-3796, https://doi.org/10.1016/j.fusengdes.2017.03.122.

[14] D. Aprile, et al., Complete compensation of criss-cross deflection in a negative ion accelerator by magnetic technique, AIP Conference Proceedings 2052, 070001 (2018); https://doi.org/10.1063/1.5083781

[15] C. J. Davisson and C. J. Calbick, Electron lenses, Phys. Rev. 38, 558 (1931); 42, 580 (1932)

[16] http://www.openmp.org 